\title{
Ocorrência de dentes fossilizados de tubarões em cascalhos biodetríticos na Praia dos Amores, Matinhos, Paraná, Brasil
}

\author{
Marcos de Vasconcellos Gernet ${ }^{1}$, Elizângela da Veiga Santos ${ }^{1,2}$ \& Esther \\ Valentina da Veiga de V. Gernet ${ }^{1}$
}

(1) Universidade Federal do Paraná, Laboratório de Ecologia Aplicada e Bioinvasões, Rua Rio Grande do Norte 145, Mirassol 83255-000, Pontal do Paraná, Paraná, Brasil. E-mail: lmv.gernet@gmail.com

(2) Universidade Federal do Paraná, Setor Litoral, Rua Jaguariaíva 512, Caiobá 83260-000, Matinhos, Paraná, Brasil. E-mail: lizveiga.ga@gmail.com

Gernet M.V., Santos E.V. \& Gernet E.V.V.V. (2018) Ocorrência de dentes fossilizados de tubarões em cascalhos biodetríticos, na Praia dos Amores, Matinhos, Paraná, Brasil. Pesquisa e Ensino em Ciências Exatas e da Natureza, 2(2): 238-241. http://dx.doi.org/10.29215/pecen.v2i2.1072

Editor acadêmico: Rodrigo Giesta Figueiredo. Recebido: 09 Julho 2018. Aceito: 22 Novembro 2018. Publicado: 28 Dezembro 2018.

Resumo: A planície costeira do Paraná originou-se no Período Quaternário a partir de movimentos cíclicos de transgressão e regressão marinha provenientes das oscilações climáticas do Holoceno. Os sedimentos da planície costeira apresentam datação que varia do Pleistoceno Superior ao Holoceno. Ao longo do litoral paranaense, são encontrados diversos pontos que apresentam depósitos de cascalho biodetrítico, com grande acúmulo de material fossilífero, proveniente deste período geológico, dentre eles dentes de tubarão. O local escolhido para realização das coletas dos dentes fossilizados foi no banco de cascalhos localizado em frente à Ilha do Farol, situada na Praia dos Amores, município de Matinhos, Paraná. Ao todo foram encontrados 563 dentes fossilizados de tubarão, de cinco diferentes espécies pertencentes a duas famílias. Estes dados são muito importantes para melhor compreendermos esta biodiversidade pretérita.

Palavras chave: Dentes de tubarão, fósseis, Quaternário, Litoral do Paraná.

Occurrence of fossilized teeth of sharks in biodetritic gravels at Praia dos Amores, Matinhos, Paraná, Brasil

Abstract: The coastal plain of Paraná originated in the Quaternary Period from cyclical movements of transgression and marine regression from the Holocene climatic oscillations. The sediments of the coastal plain present dates that vary from Pleistocene to Holocene. Along the coast of Paraná, several points are found that present deposits of biodetritic gravel, with great accumulation of fossiliferous material coming from this geologic period, among them shark teeth. The site chosen for collecting the fossilized teeth was in the gravel bank located in front of the Farol Island, located in the Amores Beach, in the municipality of Matinhos, Paraná. In total, 563 fossilized shark teeth were found, from six different species of two families. These data are very important to better understand this past biodiversity.

Key words: Shark teeth, fossils, Quaternary, coast of Paraná.

A planície costeira do Paraná originou-se no Período Quaternário a partir de movimentos cíclicos de transgressão e regressão marinha provenientes das oscilações climáticas do Holoceno (Bigarella et al. 1978). Os atuais depósitos arenosos das restingas, que se encontram comumente na forma de feixes ou cordões (Beach ridge), criam terraços que localizam-se a diferentes altitudes sobre o nível médio do mar, criando duas unidades estratigráficas distintas: a inferior, composta de areias bem estratificadas, depositadas durante o Pleistoceno, em ambiente marinho próximo à costa, e a superior, de idade Pleistocênica ou Holocênica, formada predominantemente de areias de origem eólica (Lessa et al. 2000; Angulo et al. 2005). 
De acordo com Angulo et al. (2005), os sedimentos Cenozóicos da região litorânea do Estado do Paraná foram agrupados em dois tipos principais: continentais e costeiros, com idades desde o Mioceno Inferior até o Holoceno. De acordo com o mesmo autor, os sedimentos da planície costeira com cordões litorâneos, apresentam datação que varia do Pleistoceno Superior ao Holoceno.

As formações costeiras relacionadas aos eventos marinhos transgressivos-regressivos são observadas em várias áreas litorâneas brasileiras, como citado por Buchmann \& Rincón (1997) para o Rio Grande do Sul. A presença de depósitos carbonáticos bioclásticos (concheiros ou cascalhos) representa à dinâmica destas antigas linhas de costa, possibilitando interpretar a biodiversidade pretérita (Brognon \& Verrier 1958; Boomer et al. 2003; Fulgosi et al. 2009).

Ao longo do litoral paranaense são encontrados diversos pontos que em período de maré baixa tendem a apresentar estes depósitos de cascalho biodetrítico, com grande acúmulo de material fossilífero como valvas de moluscos, equinodermos, crustáceos e dentes de tubarão, estes últimos, objetos de estudo desse trabalho. Pertencente à classe dos Chondrichthyes, os tubarões são um grupo taxonômico amplo que engloba vertebrados aquáticos mandibulados de esqueleto cartilaginoso, sendo seus dentes as estruturas mais propensas à fossilização (Compagno 1990).

O local escolhido para a realização das coletas dos dentes fossilizados foi no banco de cascalhos que se espalha por uma área de $50 \mathrm{~m}^{2}$, em frente à Ilha do Farol (2551'07" S, 48 $32^{\prime} 09^{\prime \prime}$ W) (Figura 1), também conhecida como Ilha da Tartaruga, localizado na Praia dos Amores, município de Matinhos, Estado do Paraná.

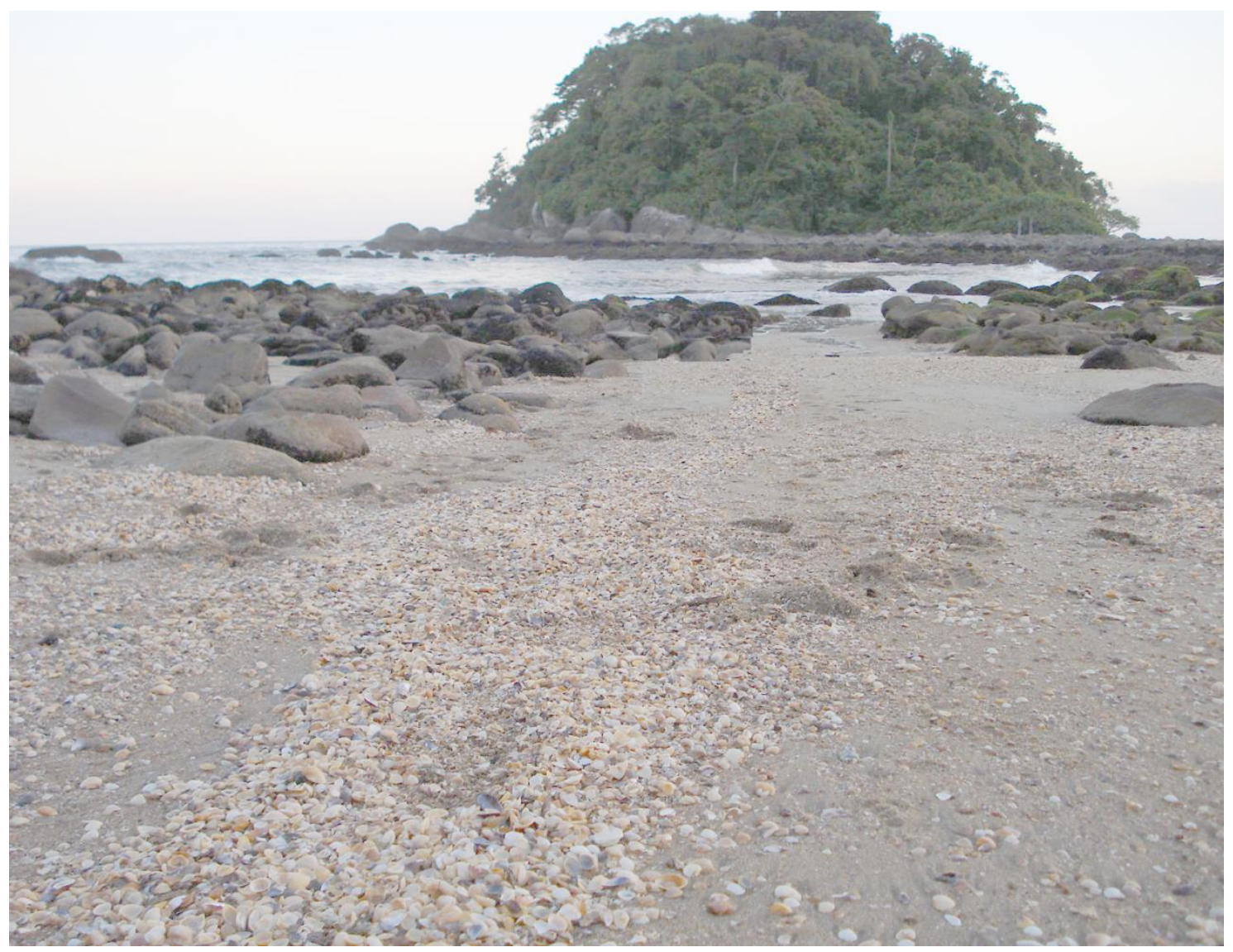

Figura 1. Depósitos de sedimentos biodetríticos em frente à Ilha do Farol, Praia dos Amores, Matinhos, Paraná.

Foram efetuadas periodicamente, prospecções visuais seguidas de coletas manuais durante dois anos, sempre em períodos de maré baixa, quando o material se encontrava exposto. 
A identificação taxonômica dos dentes de tubarão foi feita utilizando-se de literatura especializada (Cappetta 1970; Compagno 2001; Antunes \& Balbino 2004; Compagno et al. 2005).

Ao todo foram coletados 563 dentes de tubarão, de cinco diferentes espécies pertencentes a duas famílias (Tabela 1; Figura 2). A espécie Carcharodon taurus (Rafinesque, 1810) (Mangona) foi a mais abundante, corroborando com as informações do trabalho de Leavesley (2007). Em segundo lugar Carcharodon carcharias (Linnaeus, 1758) (Tubarão Branco) podendo-se inferir que em tempos passados esta espécie era mais abundante que nos dias de hoje. Galeocerdo cuvier (Péron \& Lesueur, 1822) (Tubarão Tigre) foi a espécie menos significativa, tendo sido encontrado apenas nove exemplares. Outras duas espécies identificadas foram Prionace glauca (Linnaeus, 1758) (Tintureira) e Carcharhinus brevipinna (Müller \& Henle, 1839) (Tubarão Galha Preta). Na sua maioria, os dentes encontravam-se bastante escurecidos (oxidados), bem preservados, apresentando todas as suas características.

Através destes dados, percebemos que a diversidade de espécies atuais é a mesma que a de tempos passados, variando apenas a abundância, o que pode ser explicado pela superexploração atual dos recursos pesqueiros, que tem impacto direto na diminuição das populações das espécies de tubarões encontradas atualmente na região.
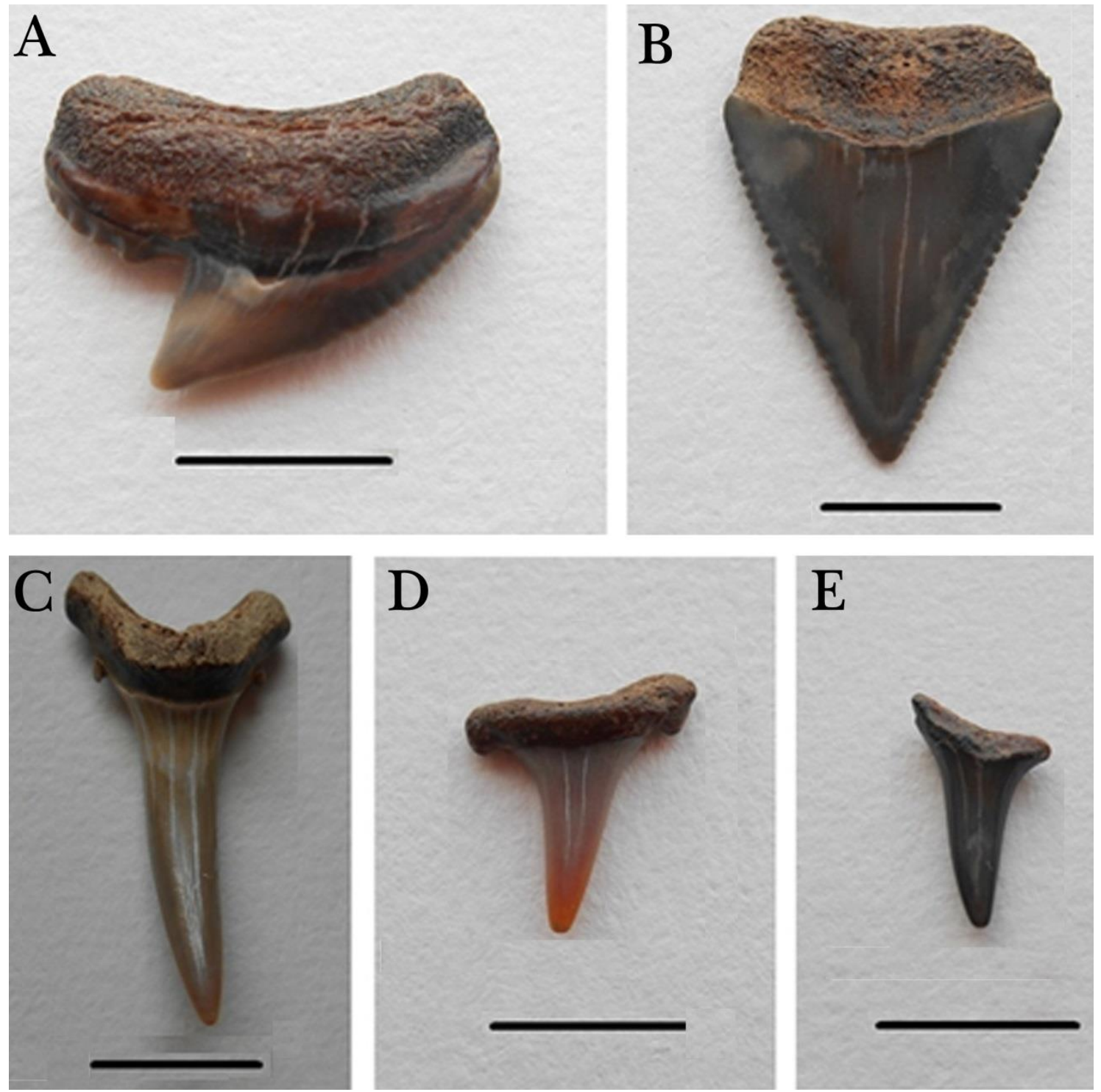

Figura 2. Dentes fossilizados das espécies de tubarões encontrados em cascalhos biodetríticos na Praia dos Amores, Matinhos, Paraná. A. Galeocerdo cuvier, B. Carcharodon carcharias; C. Carcharodon taurus; D. Prionace glauca; E. Carcharhinus brevipinna. Escala: $1 \mathrm{~cm}$. 
Tabela 1. Espécies encontradas com o número total de exemplares coletados na Praia dos Amores, Matinhos, Paraná.

\begin{tabular}{cllcc}
\hline Exemplares & \multicolumn{1}{c}{ Família } & \multicolumn{1}{c}{ Espécie } & N $^{\mathbf{0}}$ de dentes & $\%$ \\
\hline A & Carcharhinidae & Galeocerdo cuvier & 09 & 1.6 \\
B & Odontaspididae & Carcharodon carcharias & 176 & 31.3 \\
C & Odontaspididae & Carcharodon taurus & 289 & 51.3 \\
D & Carcharhinidae & Prionace glauca & 52 & 9.2 \\
E & Carcharhinidae & Carcharhinus brevipinna & 37 & 6.6 \\
\hline
\end{tabular}

\section{Agradecimentos}

Aos avaliadores anônimos pela revisão crítica do manuscrito.

\section{Referências}

Angulo R.J., Souza M.C. de, Reimer P.J. \& Sasaoka S.K. (2005) Reservoir effect of southern and southern-east Brazilian coast. Radiocarbon, 47(1): 1-7.

Antunes M.T. \& Balbino A.C. (2004) Os Carcharhiniformes (Chondrichthyes, Neoselachii) da Bacia de Alvalade (Portugal). Revista Española de Paleontología, 19(1): 73-92.

Bigarella J.J., Becker R.D., Matos D.J. \& Werner A. (1978) A Serra do Mar e a Porção Oriental do Estado do Paraná - Um problema de segurança ambiental e nacional. Curitiba: Secretaria de Estado do Planejamento e Associação de Defesa e Educação Ambiental. 249 p.

Boomer I., Horne D.J. \& Slipper I.J. (2003) The use of ostracods in Palaeoenvironmental studies, or what can you do with an ostracod Shell. Paleontological Society Papers, 9: 1-28.

Brognon G. \& Verrier G. (1958) Note sur la stratigraphie du bassin du Cuanza en Angola. Boletim da Sociedade Geológica de Portugal, 12: 61-74.

Buchmann F.S.C. \& Rincón G. (1997) Fósseis de vertebrados marinhos do Pleistoceno Superior na porção sul da planície costeira do Rio Grande do Sul, Brasil. Notas Técnicas, 10: 7-16.

Cappetta H. (1970) Les Sélaciens du Miocène de la région de Montpellier. Paleovertebrata. Montpellier: Memoire extraordinaire. 139 p.

Compagno L.J.V. (1990) Alternative life-history styles of cartilaginous fishes in time and space. Environmental Biology of Fishes, 10: 33-75.

Compagno L.J.V. (2001) Sharks of the world. An annotated and illustrated catalogue of shark species known to date. Volume 2. Bullhead, mackerel and carpet sharks (Hetero-dontiformes, Lamniformes and Orectolobiformes). Roma: FAO. 269 p.

Compagno L.J.V., White W.T. \& Last P.R. (2005) Description of a new species of weasel shark, Hemigaleus australiensis n. sp. (Carcharhiniformes: Hemigaleidae) from Australian waters. Zootaxa, 17: 37-49.

Fulgosi F.C., Casati S., Orlandini A. \& Persico D. (2009) A small fossil fish fauna, rich in Chlamydoselachus teeth, from the Late Pliocene of Tuscany (Siena, central Italy). Cainozoic Research, 6: 3-23.

Leavesley M.G. (2007) A shark-tooth ornament from Pleistocene Sahul. Antiquity, 81: 308-315.

Lessa G.C., Angulo R.J., Giannini P.C.F. \& Araújo A.D. (2000) Stratigraphy and Holocene evolution of a regressive barrier in south Brazil. Marine Geology, 165(1-4): 87-108. 